\title{
Wavepacket golden rule treatment of interparticle Coulombic decay in paired quantum dots
}

\author{
Matthias Berg, 1 a) Llinersy Uranga-Piña, ${ }^{2}$ Aliezer Martínez-Mesa, ${ }^{2}$ and Annika Bande ${ }^{1}$ \\ 1) Department of Locally Sensitive and Time-Resolved Spectroscopy, \\ Helmholtz-Zentrum Berlin für Materialien und Energie GmbH, \\ Hahn-Meitner Platz 1, 14109 Berlin, Germany \\ 2) DynAMoS (Dynamical processes in Atomic and Molecular Systems), \\ Faculty of Physics, University of Havana, San Lázaro y L, CP 10400, Havana, \\ Cuba
}

(Dated: 6 January 2020)

The interparticle Coulombic decay process in paired quantum dots is studied by electron dynamics calculations. We consider a pair of Coulomb-coupled one-electron charged gallium arsenide quantum dots embedded in a nano-wire. The two-electron decay process is approximately described by a single active electron model. Within this model, we employ the time-dependent wavepacket approach to the Fermi golden rule (introduced in the context of vibrational predissociation) to calculate autoionization rates, which are compared to exact rates obtained from fully-correlated two-electron dynamics calculations. We found that the approximated decay rates agree well with the exact results in the limit of sufficiently separated quantum dots. Finally, we explore whether the short-range behavior of the new model can be further enhanced by the inclusion of local exchange effects by means of a regularization of the Coulomb-potential based on a Jastrow-Slater wavefunction. The proposed method may open a route to study interparticle Coulombic decay in more intricate systems, e.g., paired metal-nanoparticle - quantum dot systems.

Keywords: Interparticle Coulombic decay, quantum dot, electron dynamics, quantum dynamics, autoionization

\footnotetext{
a)Electronic mail: matthias.berg@helmholtz-berlin.de
} 


\section{INTRODUCTION}

The interparticle (interatomic) Coulombic decay (ICD) is a non-local decay process, whereby a system in an inner-valence ionized electronic state decays to the electronic ground state by electron emission from a neighboring site ${ }^{1}$. In the case of atomic and molecular systems in the gas phase ICD results in the creation of a pair of ions undergoing a Coulomb explosion.

ICD takes place on the ultra fast timescales of fs to ps and over long spatial ranges from $\AA$ to nm, which make ICD often competitive compared to other decay processes such as photon emission ${ }^{1}$. ICD has been studied predominately for weakly bound van der Waals systems ${ }^{1 / 5}$ 11. Importantly, ICD and related processes also occur in water ${ }^{12}$ and in bio-molecules ${ }^{16}$, where they likely play an important role for radiation damage in living cells due the creation of highly reactive low-energy electrons 317 . Another field of increasing significance is nanoscience, where ICD has been studied for helium droplets ${ }^{18 \mid 19}$ and for hollow atoms on graphene sheets 20 . Recent comprehensive reviews have been given by Averbukh et al. $\stackrel{2}{ }$, Hergenhan ${ }^{3}$ and Jahnke ${ }^{4}$.

Another class of systems for which ICD has been predicted ${ }^{21 / 22}$ and theoretically investigated in great detail is for electrons confined to paired quantum dots (PQDs) $21 \sqrt[26]{26}$ and paired quantum wells $(\mathrm{PQWs})^{27 / 28}$. As schematically depicted in figure 1(a-d), ICD in these systems involves the deexcitation of a two-level QD mediated by the Coulomb coupling to a one-level QD which is simultaneously ionized.

Clearly, the study of decay processes due to pairing of QDs is important, as they may affect QD arrays which show potential for use in opto-electronic devices and information technology applications 29 . Furthermore, it has been proposed and corroborated by theoretical calculations that ICD in PQDs (PQWs) could be exploited for highly-sensitive wavelength specific next-generation infrared (IR) photodetectors and solar cells 2122 242727. Recent electron dynamics experiments on self-assembled QDs ${ }^{30}$ may open a promising pathway towards future ICD experiments on QDs.

ICD in PQDs takes place on the picosecond timescale and over distances of tens of nanometers $\$ 2122$. Previous theoretical investigations revealed many facets of ICD in these systems, specifically:

(i) the dependence of the decay rate on the inter-QD separation and on the shape of the 
QDs21|22|24|26|31,

(ii) the existence and effect of shape resonances 27 , leading to preferential directions for electron emission as well as the dependence of the right-to-left partial decay width on the inter-QD distance ${ }^{22}$,

(iii) the excitation by laser pulses, the presence of Rabi-oscillations and the role of multiphoton processes $23125131 / 32$,

(iv) the impact of acoustic phonons $\frac{33}{}$,

(v) the role of additional ionization channels in a three-electron three-QD system ${ }^{34}$.

Furthermore, related phenomena such as the inter-Coulombic electron capture process have been studied extensively 35 .38.

The theoretical description of intra-(conduction)-band processes is typically carried out within the single-band effective mass approximation ${ }^{39}$ (EMA). In this model, lowdimensional effective confinement potentials are employed to represent QDs and only few electrons are treated explicitly. The low dimensionality of the model, compared to that of molecular systems, then allows to study the ICD process using numerically exact electron dynamics calculations, i.e., by solving the time-dependent Schrödinger equation 2226. Another route applied to ICD in PQDs and PQWs is the numerically exact calculation of decay rates using the Fermi golden rule, where the initial and final states are subject to continuum boundary conditions in complex scaling $2127 / 28$.

In this contribution, we present an alternative but approximate treatment of the electron dynamics of ICD in PQDs. Compared to the more rigorous treatments listed above, which are limited to few-electron systems, the present approach has the potential to describe ICD for many-electron systems coupled to a QD, for example paired metal-nanoparticle QD systems. However, herein we limit the investigation to PQDs as a proof of principle and to facilitate the comparison to the exact results. The present approach is based on an approximate factorization of the two-electron wavefunction, considering only the local electrostatic Coulomb potentials exerted on the outgoing electron by the other particle in one of the two bound states of the left QD, i.e., an approximation which may hold in the limit of large QD separations.

Hence, we separate the motion of both electrons and describe the dynamics of the outgoing electron on two coupled effective potential energy surfaces (built by adding the contribution of the local interaction with the two states of the left QD and the confinement potential). 
Since the two effective potentials are energetically separated by their respective orbital energies, the initially populated one-level QD bound state on the excited state surface lies within the continuum states of the lower surface, see figure 1 (e, f). ICD as a Coulombmediated process is then invoked by considering the coupling of the two surfaces via the local transition Coulomb matrix elements. We therefore employ an effective single active electron (SAE) model in which an autoionization (ICD) process is described via the calculation of the energy dependence of the ICD width $(\Gamma(E))$ in the framework of the highly-efficient time-dependent wavepacket approach to the Fermi golden rule 40 .

We further critically compare the inter-QD distance dependence of the resonant decay rate $\Gamma$ obtained within the present approximation to accurate two-electron dynamics re-

sults obtained with the previously reported methodology 2212426. We show that the present approximation yields reliable rates for well-separated quantum dots. Close analysis of the inter-QD distance dependence of $\Gamma(E)$ reveals the presence of an irregular structure with peaks and valleys responsible for the oscillatory behavior of the resonant ICD rate with the inter-QD distance. To improve the behavior of the model at short inter-QD distances, we explore the use of effective Coulomb potentials derived from a optimized Jastrow-Slater wavefunction.

The paper is organized as follows. In Section II we describe the model system and the underlying theory. The details of the numerical calculations are given in Section III. In Section IV we present and analyze the results. Finally a summary and outlook is given in Section V.

\section{THEORY}

We consider a two-electron PQD model system, where the conduction band electronic motion is one-dimensional, representing singly-charged QDs in a GaAs nanowire ${ }^{22|23| 35 \mid 36}$ (see figure 1(a)) and described within the single-band EMA 22 26/35|36. Within the EMA, only few electrons are treated explicitly. The electronic structure of the bulk material is accounted by the effective electronic mass $m^{*}$ and by the relative static permittivity $\epsilon_{r}$ which screens the Coulomb interaction between the explicitly treated electrons. Throughout, we employ the following parameters specific to GaAs, $m^{*}=0.063 m_{e}$ and $\epsilon_{r}=12.9^{41}$. 


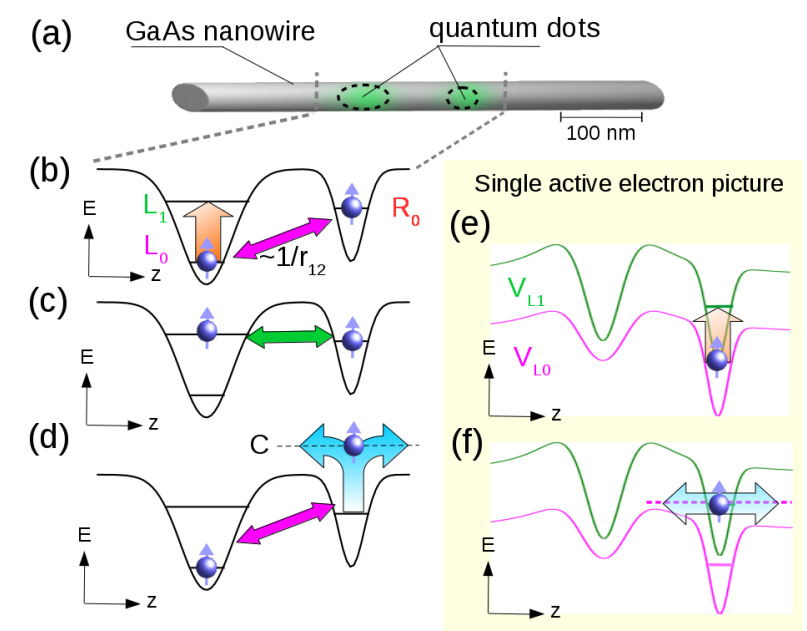

FIG. 1. Sketch of the intraband ICD process (b-d) in PQDs embedded within a GaAs nanowire (a). The electron in the left two-level QD couples through the Coulomb interaction (denoted by double arrows) to a second electron bound in the right one-level QD. The different magnitudes of the Coulomb interaction with respect to the ground and the excited state of the left QD are color coded by magenta and green, respectively. (b) The left QD is excited resonantly from the ground state. The meta-stable two-electron state (c) then decays to the final state (d) where the left QD is deexcited and the right QD ionized. (e, f) Single active electron picture of ICD in PQDs. The green (magenta) colored coupled effective potential includes the local Coulomb interaction with respect to the ground (excited) state of the left QD. (e) Excitation to the upper effective potential. (f) The electron decays into the continuum states of the lower potential. In order to facilitate the comparison of the two and one-electron pictures, Coulomb arrows in panels (b-d) and their effective potentials in panels (e-f) share the same colors. Furthermore similar colored process arrows depict excitation and ionization processes.

The two-electron Hamiltonian is given by,

$$
\hat{H}\left(z_{1}, z_{2}\right)=\hat{h}\left(z_{1}\right)+\hat{h}\left(z_{2}\right)+V_{\text {Coul. }}\left(z_{12}\right)
$$

where the one-electron Hamiltonians,

$$
\hat{h}\left(z_{i}\right)=-\frac{\hbar^{2}}{2 m^{*}} \frac{\partial^{2}}{\partial z_{i}^{2}}+V_{\mathrm{QD}}\left(z_{i}\right),
$$

contain the spatial confinement potential $V_{\mathrm{QD}}(z), V_{\mathrm{Coul}}\left(z_{12}\right)$ denotes the Coulomb interaction (further described below), $z_{i}$ and $z_{12}=\left|z_{1}-z_{2}\right|$ denote the spatial coordinates of the electrons and the inter-electronic distance, respectively. 


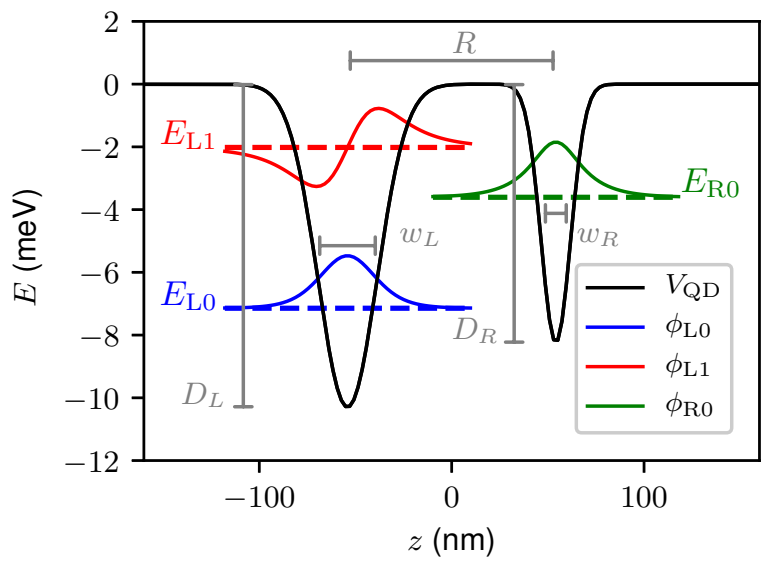

FIG. 2. The PQDs confinement potential $V_{\mathrm{QD}}$, depicted for $R=108 \mathrm{~nm}$, supports the three superimposed one-electron bound states, $\phi_{L 0}, \phi_{R 0}$ and $\phi_{L 1}$.

The spatial electronic confinement within the conduction band is described by the potential:

$$
V_{\mathrm{QD}}(z)=-D_{L} e^{-b_{L}(z+R / 2)^{2}}-D_{R} e^{-b_{R}(z-R / 2)^{2}},
$$

with inter-QD distance $R$, well depths $D_{L}$ and $D_{R}$ and width parameters $b_{L, R}=4 \ln (2) w_{L, R}^{-2}$, where $w_{L, R}$ denote the full widths at half maximum. To facilitate comparisons, we use the same confinement parameters as in Ref. ${ }^{22}$, i.e., $D_{L}=10.30 \mathrm{meV}, D_{R}=8.24 \mathrm{meV}$, and $w_{L}=36 \mathrm{~nm}, w_{R}=18 \mathrm{~nm}$.

Solving the time-independent Schrödinger equation (TISE) for the Hamiltonian given in equation (2) yields three one-electron bound states, namely $\phi_{L 0}$ and $\phi_{L 1}$ located in the left $\mathrm{QD}$, and $\phi_{R 0}$ in the right $\mathrm{QD}$, and their respective energies $E_{L 0}, E_{L 1}$ and $E_{R 0}$, as depicted in figure 2. Neglecting the Coulomb interaction at this point, the system set-up supports ICD (upon resonant excitation of the state $\phi_{L 1}$ ) by fulfilling the energy criterion $\left|E_{L 1}-E_{L 0}\right| \geq\left|E_{R 0}\right|$.

\section{A. Coulomb Interaction}

The form of the Coulomb-interaction $\sim 1 / z_{12}$ may pose problems in numerical calculations, owing both to the singularity at the origin and its long-range nature. The first of these issues is usually tackled by introducing some kind of regularization. Here we employ two different regularized Coulomb interactions, in each case the regularization is designed 
to meet specific purposes.

In the first case, we use the effective Coulomb operator for the quasi one-dimensional confinement given by Bednarek et al. $\stackrel{42}{ }$,

$$
V_{\text {Coul. }}^{\mathrm{LA}}\left(z_{12}\right)=\kappa\left(\frac{\pi}{2}\right)^{1 / 2} \frac{1}{l} \operatorname{erfcx}\left(\frac{z_{12}}{2^{1 / 2} l}\right)
$$

with $\kappa=e^{2} / 4 \pi \epsilon_{0} \epsilon_{r}, \operatorname{erfcx}(x)=\exp \left(x^{2}\right) \operatorname{erfc}(x)$, and length $l=\left(\hbar / m^{*} \omega\right)^{1 / 2}$. The potential $V_{\text {Coul. }}^{\text {LA }}\left(z_{12}\right)$ includes as a parameter the frequency $\omega$ of an additional laterally confining harmonic potential $V_{L A}(x, y)=0.5 \omega^{2}\left(x^{2}+y^{2}\right)$ and assumes that the electrons occupy the ground state of the latter at all times. Throughout, $\hbar \omega=10.30 \mathrm{meV}$, is chosen as in Refs. $22124 \sqrt{26}$ which ensures that no lateral excitations interfere with the ICD process along the $z$-direction. Equation (4) already incorporates the $x$ and $y$ contributions of the Coulombic interaction between the electrons. Hence, we refer to equation (4) as the lateral averaged (LA) Coulomb interaction.

As an alternative, the expression

$$
V_{\text {Coul. }}^{\text {cusp }}\left(z_{12}\right)=\kappa\left[\left(\frac{d u}{d z_{12}}\right)^{2}+\frac{d^{2} u}{d z_{12}^{2}}+\frac{4 \frac{d u}{d z_{12}}+1}{z_{12}}\right],
$$

with $u\left(z_{12}\right)=\frac{z_{12}}{4\left(1+\alpha z_{12}\right)}$, can be used. The functional form of equation 5 follows from the choice of the two-body wavefunction as the product of the antisymmetrized combination of single-particle orbitals and the Jastrow factor $e^{-u}$, see Refs $\underline{43} \underline{45}$. This choice allows to impose the proper asymptotic behaviour of the energy and the wavefunction as the two particles approach each other (i.e., the cusp conditions $\frac{46}{6}$ ). The specific form of the Jastrow exponent $u\left(z_{12}\right)$ depends on whether the two-body wavefunction represents a singlet or a triplet state, hence equation (5) provides a route to partially account for exchange symmetry effects in otherwise mean-field calculations. It is worth to notice that the last term in equation (5) is exact only for short separations between the two electrons. However, since the derivatives of $u\left(z_{12}\right)$ are rapidly decaying functions of $z_{12}$, we used expression (5) for the whole range of inter-particle distances. We limit our investigation to triplet wavefunctions, thus equation (5) explicitly reads:

$$
\begin{aligned}
V_{\text {Coul. }}^{\text {cusp }}\left(z_{12}\right)=\kappa[ & -\frac{1}{16\left(1+\alpha z_{12}\right)^{4}}+\frac{\alpha}{2\left(1+\alpha z_{12}\right)^{3}} \\
& \left.+\frac{\alpha}{\left(1+\alpha z_{12}\right)^{2}}+\frac{\alpha}{\left(1+\alpha z_{12}\right)}\right]
\end{aligned}
$$




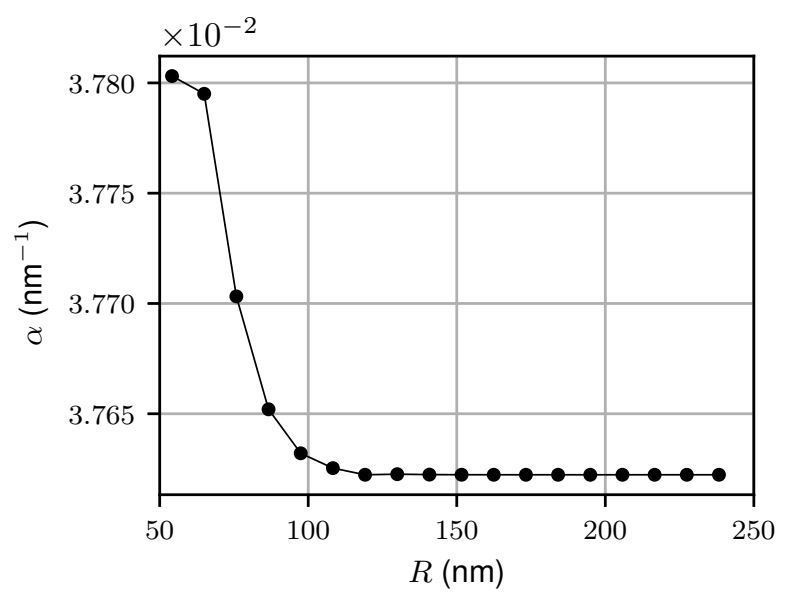

FIG. 3. Size parameter $\alpha(R)$ entering in the definition of the Jastrow factor, as a function of the distance $R$ between the quantum dots.

In Quantum Monte Carlo calculations, it is customary to obtain the values of the variational parameters entering in the definition of the Jastrow factor via the optimization of the expectation value of the ground state energy 43147 . Here we follow a different route, because the parameter $\alpha$ is required to mimic the role of two-body correlations in dynamical phenomena such as the decay rate. Specifically, the value of $\alpha$ is chosen by imposing that the size of the "exchange hole" in the resulting pair correlation function matches the one calculated within the Hartree-Fock approximation for spin-parallel electrons in one dimension 43 . It can be seen, that this procedure results in a function $\alpha(R)$ which is only weakly dependent on the separation $R$ between the quantum dots (variations are lower than 1\%, cf. figure 3 ). It suggests that a single value of $\alpha$ suffices to capture the effect of both short-range and long-range electron correlations in this problem.

Depictions of Coulomb potentials $V_{\text {Coul. }}\left(z_{12}\right)$ are given in figure 4. Both regularization schemes lead to a sizable attenuation of the Coulomb interaction at short distances, which is more pronounced in the case of cusp compared to LA regularization, respectively.

\section{B. ICD rate from two-electron dynamics}

Within this investigation, we employ the exact numerical electron-dynamics treatment, given in Refs. ${ }^{22|24| 26}$ to calculate the reference ICD rates. We numerically solve the time- 


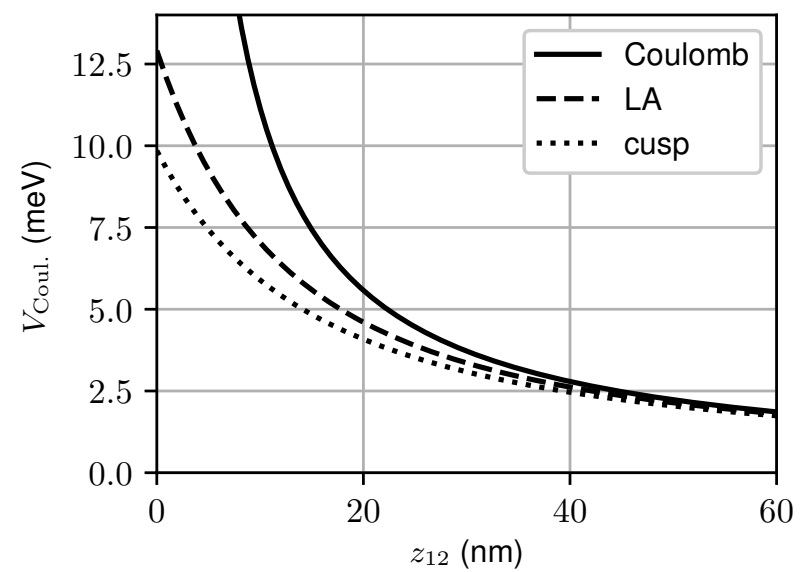

FIG. 4. Regularized Coulomb potentials $V_{\text {Coul. }}^{\text {reg. }}\left(z_{12}\right)$ obtained by taking into account either lateral confinement (LA, dashed line), or by obeying the cusp condition (cusp, dotted line, $\alpha=0.03762$ $\mathrm{nm}^{-1}$ ). The latter yields a less repulsive effective potential, although both choices of $V_{\text {Coul. }}^{\text {reg. }}$ approach the non-regularized Coulomb potential (full line) as $z_{12}$ increases.

dependent Schrödinger equation,

$$
i \hbar \frac{\partial}{\partial t} \Psi\left(z_{1}, z_{2}, t\right)=\hat{H}\left(z_{1}, z_{2}\right) \Psi\left(z_{1}, z_{2}, t\right)
$$

where the antisymmetrization of the spatial two-electron spin-triplet wavefunction, $\Psi\left(z_{1}, z_{2}, t\right)=$ $-\Psi\left(z_{2}, z_{1}, t\right)$ is imposed. To determine the ICD rate, the initial two-electron L1R0 state, i.e., with the electron configuration $\Psi\left(z_{1}, z_{2}, t=0\right)=\left[\phi_{L 1}\left(z_{1}\right) \phi_{R 0}\left(z_{2}\right)-\phi_{L 1}\left(z_{2}\right) \phi_{R 0}\left(z_{1}\right)\right]$, is propagated. Although L1R0 can be obtained from a bound state calculation (within an $\mathcal{L}^{2}$ basis), it is actually a meta-stable state, that is, it is energetically degenerate with a state of the configuration L0C, where C denotes a continuum state. During the propagation $\Psi\left(z_{1}, z_{2}, t=0\right)$ therefore decays towards L0C and the respective decay width $\Gamma$ is obtained from an exponential fit to the absolute squared autocorrelation function:

$$
\left|\left\langle\Psi\left(z_{1}, z_{2}, t=0\right) \mid \Psi\left(z_{1}, z_{2}, t\right)\right\rangle\right|^{2} \propto e^{-\Gamma t}
$$

Equivalently, the decay process can be characterized by the lifetime $\tau=\hbar / \Gamma$. In the case of the two-electron calculations, we employ the LA Coulomb interaction $V_{\text {Coul. }}^{\text {LA }}\left(z_{12}\right)$, equation (4). 


\section{ICD rate from the single active electron time-dependent Fermi golden rule (TDFGR) approach}

In the remainder of this section, we describe the time-dependent framework for the approximate evaluation of the decay rates for ICD electron dynamics in PQDs. Within the approximation, we compare results obtained for the two regularization approaches for the Coulomb interaction in the PQDs system:

(a) the electrons are only weakly correlated and thus the exchange correlation can be neglected completely. Hence we employ $V_{\text {Coul. }}^{\mathrm{LA}}\left(z_{12}\right)$, and

(b) the partial inclusion of the effects of the exchange symmetry within a mean field description using Jastrow functions through $V_{\text {Coul. }}^{\text {cusp }}\left(z_{12}\right)$.

By comparing the results of the simulations carried out using these two approaches, we may assess the validity of assumption (a), which may hold as long as the separation of the QDs is sufficiently large and also for the target final ICD states (the final two-electron wavefunctions are simple products of the relevant one-electron wavefunctions).

The time-dependent single active electron approximation (SAE) to ICD in PQDs is based on the integration of the two-particle wavefunction over the coordinate of the electron that remains bound. Hence, the probability density distribution of the outgoing electron is given by $\rho\left(z_{2}, t\right)=\int d z_{1}\left|\Psi\left(z_{1}, z_{2}, t\right)\right|^{2}$. Analogously, we define effective Hamiltonians for the initial and final channels of the ionization dynamics of the single active electron as:

$$
\hat{H}_{\lambda} \psi\left(z_{2}, t\right)=\int d z_{1} \frac{\Psi^{*}\left(z_{1}, z_{2}, t\right) \hat{H} \Psi\left(z_{1}, z_{2}, t\right)}{\left[\psi\left(z_{2}, t\right)\right]^{*}},
$$

where $\psi\left(z_{2}, t\right)$ is the wavefunction describing the state of the emitted electron, and $\lambda=$ L0, L1.

In equation (9), the two-electron wavefunction is modeled either as a factorized product of single-particle functions, $\phi_{\lambda}\left(z_{1}, t\right) \psi\left(z_{2}, t\right)$, or an antisymmetrized linear combination of these single-particle orbitals times the Jastrow factor

(i.e., $\left.\Psi\left(z_{1}, z_{2}, t\right)=\frac{1}{\sqrt{2}}\left[\phi_{\lambda}\left(z_{1}, t\right) \psi\left(z_{2}, t\right)-\phi_{\lambda}\left(z_{2}, t\right) \psi\left(z_{1}, t\right)\right] e^{-u}\right)$. These two cases correspond, respectively, to approximations (a) and (b), as introduced at the beginning of this section (II C).

The integration of equation (1) over the ground and excited states of the left $\mathrm{QD}, \phi_{\mathrm{L} 1}\left(z_{1}\right)$ 
and $\phi_{\mathrm{L} 0}\left(z_{1}\right)$, yields

$$
\hat{H}_{L 1}=\left\langle\phi_{L 1}\left(z_{1}\right)|\hat{H}| \phi_{L 1}\left(z_{1}\right)\right\rangle=-\frac{\hbar^{2}}{2 m^{*}} \frac{\partial^{2}}{\partial z_{2}^{2}}+V_{L 1}\left(z_{2}\right),
$$

and

$$
\hat{H}_{L 0}=\left\langle\phi_{L 0}\left(z_{1}\right)|\hat{H}| \phi_{L 0}\left(z_{1}\right)\right\rangle=-\frac{\hbar^{2}}{2 m^{*}} \frac{\partial^{2}}{\partial z_{2}^{2}}+V_{L 0}\left(z_{2}\right)
$$

respectively.

This treatment allows to describe effectively the dynamics of the electron being emitted from the right $\mathrm{QD}$, by being subjected to the electrostatic potential of the other electron occupying the state $\phi_{L 0}$ or $\phi_{L 1}$. Therefore, the time evolution of the single-active electron wavepacket takes place on two coupled effective potentials, $V_{L 0}, V_{L 1}$ given by

$$
V_{L 0}=E_{L 0}+V_{Q D}\left(z_{2}\right)+\left\langle\phi_{L 0}\left(z_{1}\right)\left|V_{\text {Coul. }}\left(z_{12}\right)\right| \phi_{L 0}\left(z_{1}\right)\right\rangle
$$

and

$$
V_{L 1}=E_{L 1}+V_{Q D}\left(z_{2}\right)+\left\langle\phi_{L 1}\left(z_{1}\right)\left|V_{\text {Coul. }}\left(z_{12}\right)\right| \phi_{L 1}\left(z_{1}\right)\right\rangle
$$

where the last terms denote the averaged electrostatic potentials weighted by the single particle bound states of the two-level QD.

Furthermore, within the SAE approximation, the ionization is induced by the Coulomb matrix element $\hat{W}$ for the $\phi_{L 1}$ to $\phi_{L 0}$ transition given by

$$
\hat{W}=\left\langle\phi_{L 0}\left(z_{1}\right)\left|V_{\text {Coul. }}\left(\left|z_{2}-z_{1}\right|\right)\right| \phi_{L 1}\left(z_{1}\right)\right\rangle .
$$

The SAE approximation to ICD in PQDs thus describes the motion of the outgoing electron on two effective potentials $V_{L 0}$ and $V_{L 1}$ which are coupled by $\hat{W}$ and include the static Coulomb barriers of the L0 and L1 states of the electron in the left QD.

Depictions of the effective potentials and of the matrix elements $\hat{W}$ for a typical paired QD set-up are given in figure 4 and in figure 5, respectively. In figure 5 it is worth to notice that the more attenuated cusp regularized Coulomb interaction results in lower Coulomb barriers and deeper wells compared to the regularization via lateral averaging. These features will be further reflected on the behavior of the corresponding decay rates.

The SAE ICD rates $\tilde{\Gamma}$ can be calculated using the Fermi golden rule:

$$
\tilde{\Gamma}=\frac{2 \pi}{\hbar}\left|\left\langle\phi_{R}^{V L 0}\left(z_{2}\right)|\hat{W}| \phi_{C}\left(z_{2}\right)\right\rangle\right|^{2}
$$




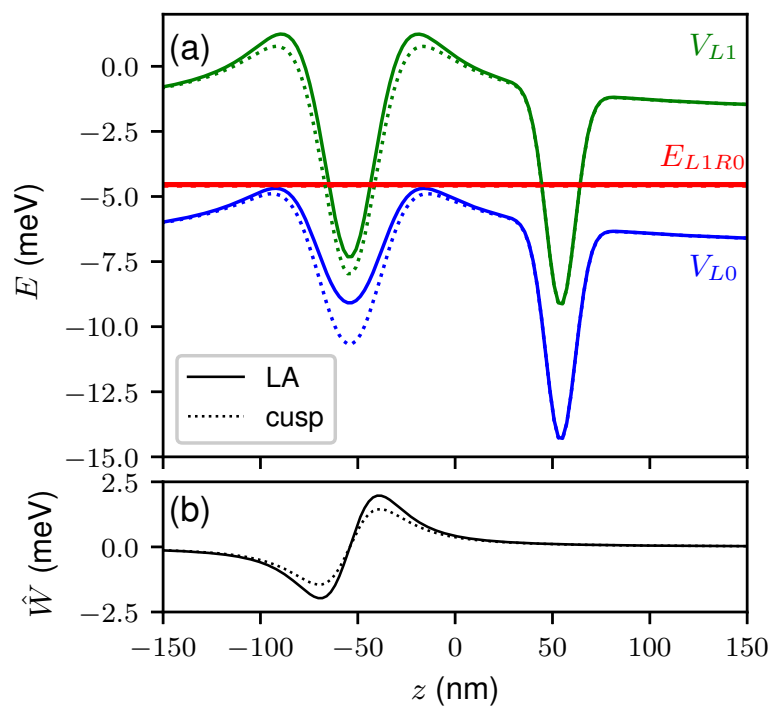

FIG. 5. (a) The SAE, with resonance energy $E_{L 1 R 0}$, moves on coupled effective potentials. Initially bound within the right hand side of $V_{L 1}$, it decays to the continuum states of $V_{L 0}$ coupled by the $\mathrm{L} 1 \rightarrow \mathrm{L} 0$ transition electrostatic potential $\hat{W}$ shown in panel (b). The more attenuated cusp regularized Coulomb interaction (dotted lines) results in lower Coulomb barriers and deeper wells, compared to the regularization via LA (full lines).

where the integration is carried out over the coordinate of the outgoing electron. The initial state $\psi(z, t=0)=\phi_{R}^{V L 0}\left(z_{2}\right)$ is determined by solving the TISE for equation (11), i.e., with respect to the effective potential $V_{L 0}\left(z_{2}\right)$, and choosing the bound state which is localized in the right well. The final continuum state $\phi_{C}\left(z_{2}\right)$ depends on the energy $E_{L 1 R 0}$ of the excited ICD resonance. The photoexcitation is assumed to be instantaneous, hence the wavepacket promoted onto the excited potential energy surface is the exact copy of the ground state of the right QD. Within the SAE model, this procedure formally translates into placing $\phi_{R}^{V L 0}\left(z_{2}\right)$ on the effective potential $V_{L 1}\left(z_{2}\right)$ of the excited state L1, whereas $E_{L 1 R 0}$ is approximated by the energy $E_{R}^{V L 1}$ of the bound state $\phi_{R}^{V L 1}\left(z_{2}\right)$ of the effective potential $V_{L 1}\left(z_{2}\right)$.

Upon definitions of the Hamiltonians of the initial and final states (equations (10) and (11), respectively), and the transition electrostatic potential (equation (14)), the ICD process can be monitored by following the time evolution of the wavefunction of the single active electron. The initial electronic wavepacket $\phi_{R}^{V L 0}$ on $V_{L 1}$ is coupled via $\hat{W}$ to the continuum states of $V_{L 0}$. Therefore, we calculate $\tilde{\Gamma}$ for the SAE model using the time-dependent 
wavepacket version of the Fermi golden rule ${ }^{40}$ (TDFGR) via the propagation of the initial state $\Phi_{0}=\hat{W} \phi_{R}^{V L 0}$ on the final dissociative surface (that is, solving the time-dependent Schrödinger equation using the Hamiltonian $\left.\hat{H}_{L 0}\right)$. Although in general $\Phi_{0}$ lacks a direct physical meaning, for the specific case of ICD in paired QDs this effective wavepacket resembles the time evolution of the reduced single particle density $\rho\left(z_{2}, t\right)$ within a mean field approximation. This feature is further elaborated in the Results section. The decay rates evaluated within the wavepacket approach were found to be in very close agreement with those obtained using the time-independent version of the Fermi golden rule (equation (15)).

As shown in Ref. ${ }^{40}$, the decay width $\tilde{\Gamma}$ can be computed either:

- from the autocorrelation function of the wavepacket $\left\langle\Phi_{0} \mid \Phi(t)\right\rangle$ with $\Phi(t)=\mathrm{e}^{-i \hat{H}_{L 0} t / \hbar} \Phi_{0}$ (see equation (6) in Ref. $\stackrel{40}{ })$, or

- from the autocorrelation function of the projection $\Phi_{c}\left(t_{0}\right)$ of the wavepacket $\Phi_{0}$ onto the continuum eigenstates of the Hamiltonian $\hat{H}_{L 0},\left\langle\Phi_{c}\left(t_{0}\right) \mid \Phi_{c}(t)\right\rangle$, e.g.,

$$
\tilde{\Gamma}(E)=\frac{1}{2 \hbar} \int_{-\infty}^{\infty} d t e^{i E t / \hbar}\left\langle\Phi_{c}(0) \mid \Phi_{c}(t)\right\rangle .
$$

For dynamical processes taking place in the time scale of picoseconds or longer, such as ICD in PQDs, the second choice is preferred due to the faster decay of the autocorrelation function of $\Phi_{c}(t)$, and it is the method employed to compute the ICD rates presented in Section IV. In practice $\Phi_{c}(t)$ is calculated by subtraction of all bound state fractions from the initial wavepacket $\Phi_{0}$.

$$
\Phi_{c}(t)=\Phi_{0}-\sum_{\beta} c_{\beta} \phi_{\beta},
$$

where $\beta$ labels the left and right potential wells, and $c_{\beta}=\left\langle\Phi_{c} \mid \phi_{\beta}^{V L 0}\right\rangle$. It should be noted that equation 16 yields the spectrum of decay rates $\tilde{\Gamma}(E)$, where the actual decay rate has to be evaluated at the resonance energy $E=E_{R}^{V L 1}$.

\section{COMPUTATIONAL DETAILS}

\section{A. Two-electron calculations}

For two-electron dynamics the variational multi configuration time-dependent Hartree (MCTDH) method $\sqrt[48 / 49]{ }$, as implemented in the MCTDH package of programs $\sqrt{50151}$, is employed. MCTDH wavefunctions are represented as sums of products of single-particle func- 
tions (SPFs). Two-electron wavefunctions are

$$
\Psi\left(z_{1}, z_{2}, t\right)=\sum_{i} \sum_{j} A_{i j}(t) \varphi_{i}\left(z_{1}, t\right) \varphi_{j}\left(z_{2}, t\right)
$$

where $\varphi_{i}$ and $\varphi_{j}$ denote time-dependent SPFs and $A_{i j}$ are time-dependent coefficients. Spatial antisymmetry of the spin triplet two-electron wavefunctions is enforced by constricting the coefficients to $A_{i j}=-A_{i j}$. The respective MCTDH equations of motions for the coefficients $A_{i j}$ and the single particle functions $\varphi_{i}$ follow from the Dirac-Frenkel variational principle.

Wavefunctions and operators are represented on grids of 140 equally-spaced points between $-541.5 \mathrm{~nm}$ and $541.5 \mathrm{~nm}$ in the sine discrete variable representation (DVR) explicit two-electron calculations, the Coulomb potential was expanded into product form using the POTFIT algorithm $[52$. Time-independent solutions are obtained by improved-block relaxation to the lowest 52 two-electron states which were expressed by 48 SPFs per electron. In propagations 8 SPFs per electron coordinate were used. The employed grids and numbers of SPFs per electron lead to well converged dynamics as was shown in Ref. 24 . In the case of propagations of ICD dynamics, complex absorbing potentials (CAPs) of order four with a strength parameter of $10^{-5}$ at positions $\pm 324.9 \mathrm{~nm}$ were added to the Hamiltonian, in order to remove the continuum electron density from the system. Reference decay widths from two-electron dynamics were obtained by exponential fits of the absolute square of the autocorrelation function beginning from times of 16 ps. In this way, initial faster decays due to spurious continuum parts present in the initial wavefunction (see the reduced two-particle density distribution at $t=0 \mathrm{ps}$ in figure 8b) are filtered out.

\section{B. Single active electron TDFGR calculations}

Single active electron (SAE) dynamics in the framework of the TDFGR have been performed for the LA and cusp Coulomb interactions using the MCTDH package employing one SPF represented on a regular sine-DVR grid of 1000 points from -1083 nm to $1083 \mathrm{~nm}$. As absorbing boundaries, CAPs placed at $\pm 866.4 \mathrm{~nm}$ with the same parameters as in the two-electron case (see above) have been used. 


\section{RESULTS}

Before we describe in detail the results on the ICD rates determined with the SAE approximation via the TDFGR, we focus on the time-independent properties of the effective potentials underling the SAE for ICD in PQDs. The resulting effective potentials $V_{L 0}$ and $V_{L 1}$ for an inter-QD separation of $R=108 \mathrm{~nm}$ are depicted in figure 4. Focusing on the
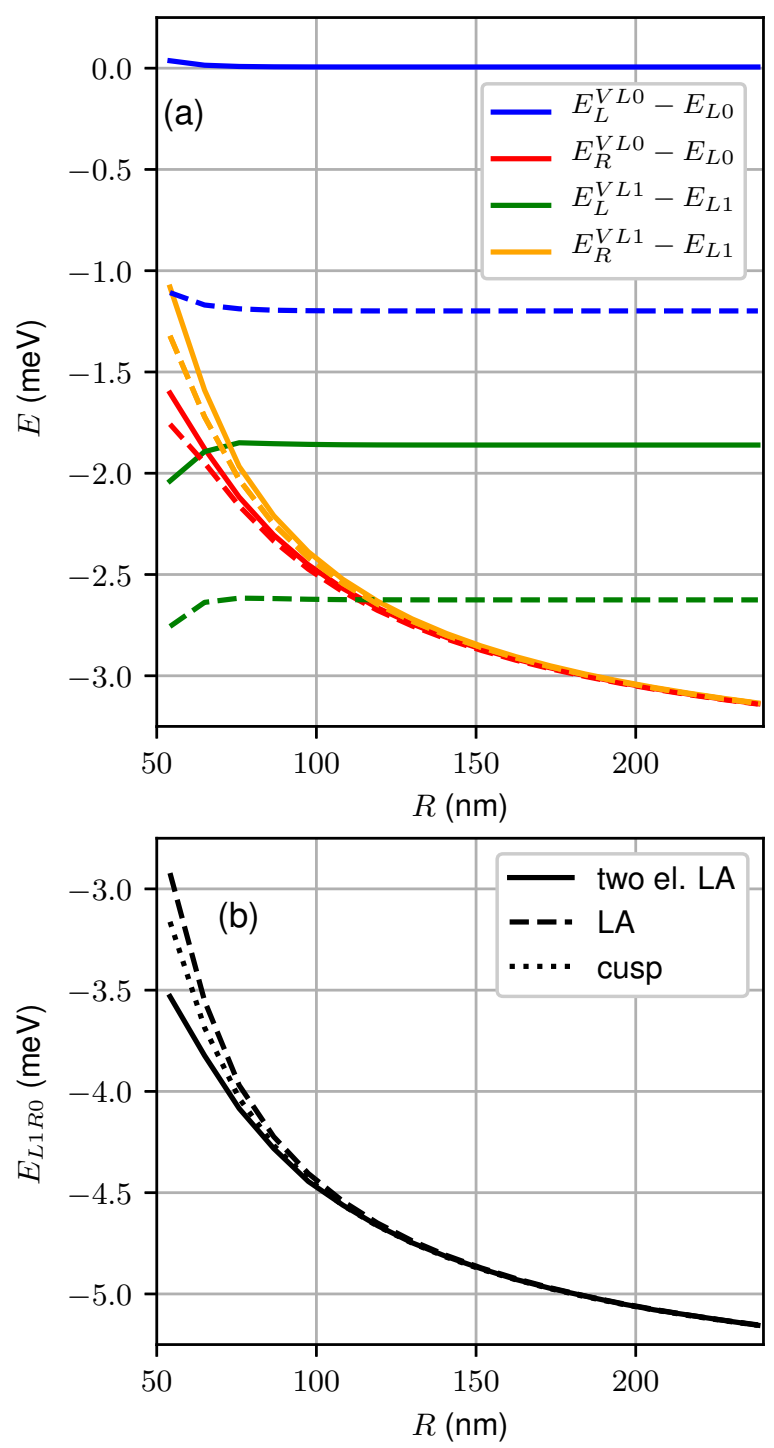

FIG. 6. (a) inter-QD distance dependence of the one-electron bound and localized state energies of the SAE effective potentials, derived from the Coulomb potentials via LA (full lines) and cusp condition (dashed lines) regularization. (b) inter-QD distance dependence of the two-electron energy of the ICD resonance state L1R0 within the two-electron and SAE approximation. 
left side of the potentials, we notice that the Coulomb barrier due to the L0 and L1 states significantly reduces the well depth of the left QD. However, the confinement potential of the right QD is only marginally affected. The Coulombic distortion of the right QD confinement reduces when the inter-QD separation is increased as the well is located in the tail of the Coulomb barrier.

This behaviour is reflected in the inter-QD distance dependence of the SAE bound state energies of both potentials, which are shown in figure 6(a). To allow a better comparison, the energies of the L0 and L1 states have been subtracted from the respective SAE bound state energies. With the increase of the inter-QD separation the energies of the SAE states $E_{R}^{V L 0}, E_{R}^{V L 1}$, localized at the right $\mathrm{QD}$, decrease due to the aforementioned reduction in the Coulomb distortion. Furthermore, differences in $E_{R}^{V L 0}, E_{R}^{V L 1}$ besides the constant energy shifts $E_{L 0}$ and $E_{L 1}$ vanish in the limit of large inter-QD separation, which begins at about $R=150 \mathrm{~nm}$. The Coulomb barriers in the present setup are not high enough to hinder the occurrence of SAE bound-states located over the left well. This is especially true for the excited state surface $V_{L 1}$ and all surfaces derived from the cusp-regularized Coulomb interaction. Since we consider a triplet two-electron system, these states should be considered as unwanted artifacts within the SAE model, and are manifestation of the neglect of proper exchange interactions. The energies $E_{L}^{V L 0}, E_{R}^{V L 1}$ of these states vary only for very short $(R<75 \mathrm{~nm})$ inter-QD distances due to the modulation of the underlying one-electron L1 and L0 bound states by the confinement potential of the right QD. Differences in the bound state energies that arise due to the choice of regularization of the Coulomb interaction, i.e., LA or cusp, are negligible for states bound in the right well, $E_{R}^{V L 0}$ and $E_{R}^{V L 1}$, and large for those bound in the left well, due to the stronger attenuation resulting from cusp regularization in comparison to LA regularization.

Within the SEA the energy $E_{R}^{V L 1}$ approximates the energy of the ICD resonance $E_{L 1 R 0}$. In figure 6(b) the variation of $E_{L 1 R 0}$ with the inter-QD distance, determined by the SEA approximations and the two-electron reference calculations, is presented. For short interQD separations $R<100 \mathrm{~nm}$ the SEA resonance energy deviates up to $+0.5 \mathrm{meV}$ from the exact value. At larger inter-QD separation $R>100 \mathrm{~nm}$ SEA results coincide with the exact results, thereby pointing to the validity of the SEA in the limit of sufficiently separated QDs. It should be noted that SEA results obtained with the cusp regularized Coulomb interaction reduce the error of the resonance energies at short inter-QD distances. 
We initially focus on the decay calculated within the single active electron TDFGR approach for a PQD system with an inter-QD separation of $R=108 \mathrm{~nm}$. The initial wavefunction of the active electron $\phi_{R}^{V L 0}$, the effective wavepacket $\Phi_{0}=\hat{W} \phi_{R}^{V L 0}$, and the projection $\Phi_{c}\left(t_{0}\right)$ of the later on the continuum states are shown in figure 7 , panels (a), (b) and (c), respectively. In the case of the effective wavepacket $\Phi_{0}$, the action of the transition Coulomb
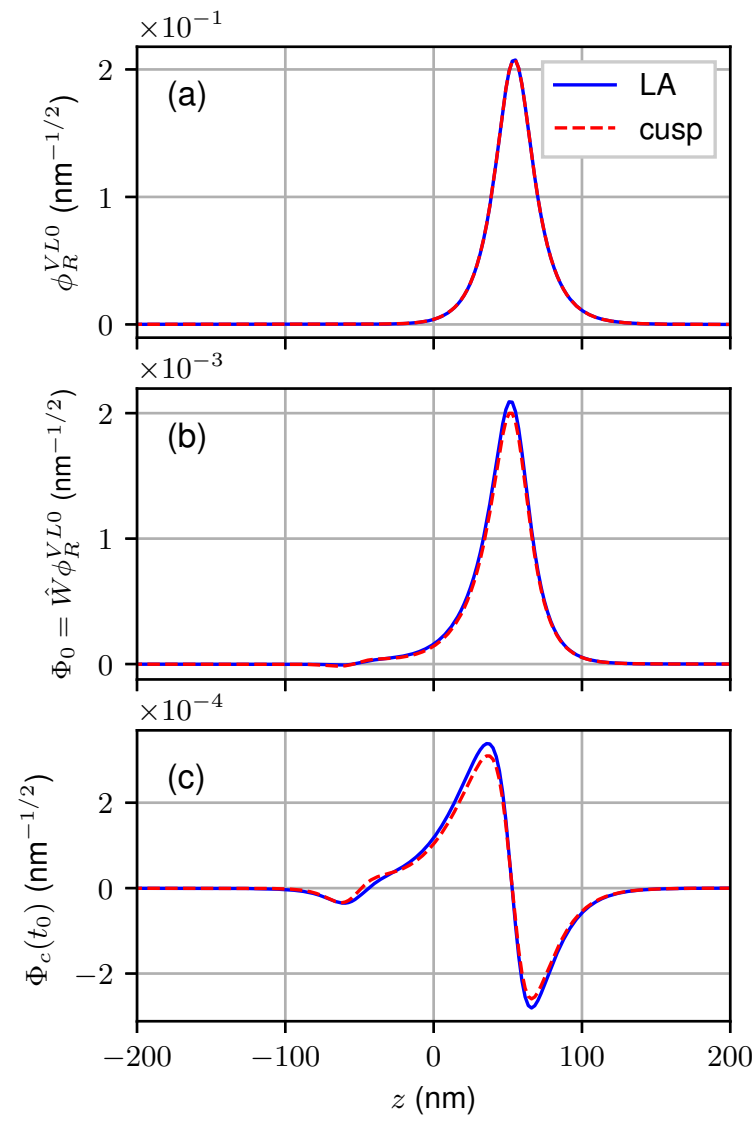

FIG. 7. Initial SAE TDFGR continuum wavepacket $\Phi_{c}\left(t_{0}\right)(\mathrm{c})$, for an inter dot separation of $R=$ $108 \mathrm{~nm}$. Panel (a) shows the bound state $\phi_{R}^{V L 0}$ that is localized on the right well of the effective potential $V_{L 0}$. Panel (b) shows the wavepacket $\Phi_{0}$ created by the action of $\hat{W}$ on $\phi_{R}^{V L 0}$. Panel (c) shows the continuum part $\Phi_{c}$ of the wavepacket in (b).

matrix element $W$ only leads to a small distortion of the shape of the SAE bound state $\phi_{R}^{V L 0}$. Consequently, a large overlap exists with the latter bound state. In spite of the fact that the action of the operator $W$ on the wavefunction $\phi_{R}^{V L 0}$ does not modify significantly the spatial dependence of the latter, it is worth stressing that the overall magnitude of $\Phi_{0}$ is two orders of magnitude smaller. This is indicative of the rate at which population is transferred from 
the initial into the final channel.

Although being conceived originally as a numerical recipe for computing the resonance width, the latter properties provide the wavepacket $\Phi(t)=e^{-i H_{L_{0}} t / \hbar} \Phi_{0}$ propagated in the present framework with physical meaning. Since $\Phi_{0}$ has a significantly large projection on the state $\phi_{R}^{V L 0}$, the time-dependent wavefunction $\Phi(t)$ is indicative of the time evolution of the actual wavepacket of the outgoing electron (within a mean field description). 


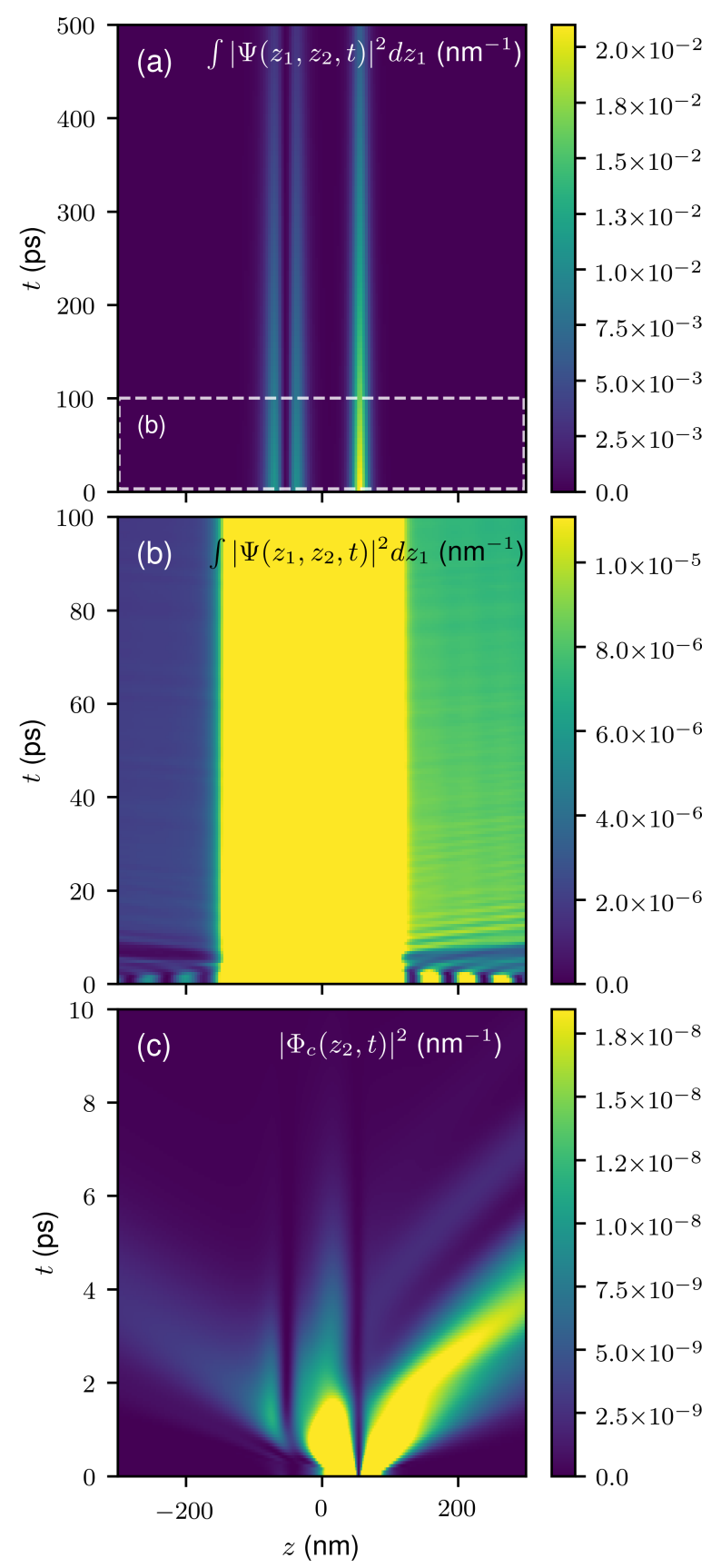

FIG. 8. Wavepacket dynamics of ICD for a PQD system with $R=108.3 \mathrm{~nm}$ comparing twoelectron (a,b) and SAE TDGFR (c) results. (a) Evolution of the reduced density obtained from the two-electron wavefunction up to $t=500$ ps. (b) Change of the scaling reveals the outgoing electron density within the continuum. (c) Evolution of the continuum wavepacket prepared according to the SAE TDFGR until $t=10$ ps. In both cases $(\mathrm{b}, \mathrm{c})$ the ICD electron leaves the PQD predominantly to the right hand side. 
This aspect is further explored in figure 8, where the time evolution of the electron density obtained from the two-electron calculation (figure $8(a, b)$ ) is displayed along with the short time evolution of the SAE density (up to $10 \mathrm{ps}$, figure $8(\mathrm{c})$ ) for the case of PQDs lying $R=$ $108 \mathrm{~nm}$ apart. To discuss the features of the two-electron results two different scales for the density are employed. The evolution of the electron density in the region of the two QDs for up to 500 ps (figure 8(a)) shows a continuous decrease of the density of the initial

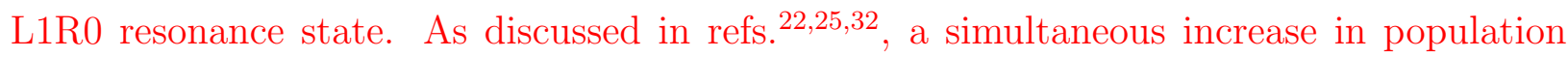
of an L0 state on the left QD is not observed as the L0C bound/continuum two-electron states are absorbed by the left and right CAPs. The evolution of the electron density in the continuum within the first $100 \mathrm{ps}$ is depicted in figure $8(\mathrm{~b})$. For $t<5 \mathrm{ps}$ a fast decay of spurious continuum parts of the initial L1R0 state is observed. The latter are a numerical artifact ${ }^{22}$, which makes it necessary to discard the first 16 ps when evaluating the ICD rate form two-electron autocorrelation functions. Outgoing electron density due to ICD is observed for $t>5 \mathrm{ps}$, and points to the existence of a preferred direction for the electron emission, more electron density leaves the system to the right hand side $(z>0)$.

The single active one-electron TDFGR dynamics yields a qualitatively similar picture of the ICD process. Indeed, the short-time evolution of the projection on the continuum states of the reduced probability density associated with the second electron (bottom panel of figure 8) also indicates that the outgoing electron moves away from the QD predominantly to the right hand side. Noteworthy, the expansion of $\left\|\Phi_{c}\left(z_{2}, t\right)\right\|^{2}$ occurs in a markedly shorter time scale compared to the exact two-electron dynamics.

The evolution of the initial state $\Phi_{0}$ on $V_{L 0}$ gives rise to a highly-oscillatory autocorrelation function (see figure 9(a)), which approaches a non zero final value. Subtraction of this boundstate population from $\Phi_{0}$ prior to propagation gives the pure continuum part of the initial wavepacket $\Phi_{c}\left(t_{0}\right)$, which decays rapidly on $V_{L 0}$. The respective autocorrelation function is presented in panel (b) of figure 9. As anticipated, the continuum dynamics within the TDFGR approach is completed within 13 ps, i.e., within a fraction of the ICD lifetime of $\tau=400$ ps determined by reference two-electron dynamics calculations. In addition to the different timescales, the decay of the autocorrelation function of the projection of the wavepacket $\Phi(t)$ on the continuum states (i.e., $\left.\left\langle\Phi_{c}\left(t_{0}\right) \mid \Phi_{c}(t)\right\rangle\right)$ is smoother, which makes the latter easier to handle numerically.

Performing the Fourier transformation on the autocorrelation function for the continuum 




FIG. 9. Autocorrelation functions for wavepacket dynamics of the decaying initial SAE state (top) and its respective continuum part (bottom) within the TDFGR on the $V_{L 0}$ surface, for an inter-QD separation of $R=108 \mathrm{~nm}$.

dynamics (equation (16)) yields the spectrum of decay widths $\tilde{\Gamma}(E)$, shown in figure 10 aligned with the effective potentials $V_{L 0}$. The results for the inter-QD distance $R=108 \mathrm{~nm}$ are presented in figure $10(\mathrm{~b})$. Evaluation of $\tilde{\Gamma}(E)$ at the energy of the bound state $\phi_{R}^{V L 1}$, $E_{R}^{V L 1}=-4.54 \mathrm{meV}$, finally gives the ICD width obtained within the single active electron TDFGR approximation of $\tilde{\Gamma}\left(E_{R}^{V L 1}\right)=8.8 \cdot 10^{-4} \mathrm{meV}$, which translates into an ICD lifetime of $\tilde{\tau}=750$ ps.

Comparing this result to the ICD width $\Gamma=1.7 \cdot 10^{-3} \mathrm{meV}$ and respective lifetime $\tau=400$ ps obtained from the exact two-electron dynamics calculations shows that the single active electron TDFGR approximation underestimates the ICD width by a factor of two. While this difference seems significant at first, it is worth noting that for the chosen separation between the QDs, the resonance energy is very close to the height of the energy barriers in the vicinity of the left QD for the final dissociative potential energy surface. The transmission probability across this double barrier (and consequently, the decay width) is very sensitive to small variations of the effective potentials, thereby providing a stringent test for the single active electron TDFGR approach. For example, the choice of the cusp regularization condition results in slightly lower Coulomb barriers, compared to LA regularization. This changes the interaction of the ICD electron with the double barrier, yielding $\tilde{\Gamma}(E)$ spectra which are significantly modulated at energies close to the barrier height, as depicted in figure 10 for three different inter-QD distances. As it is shown in the following, 

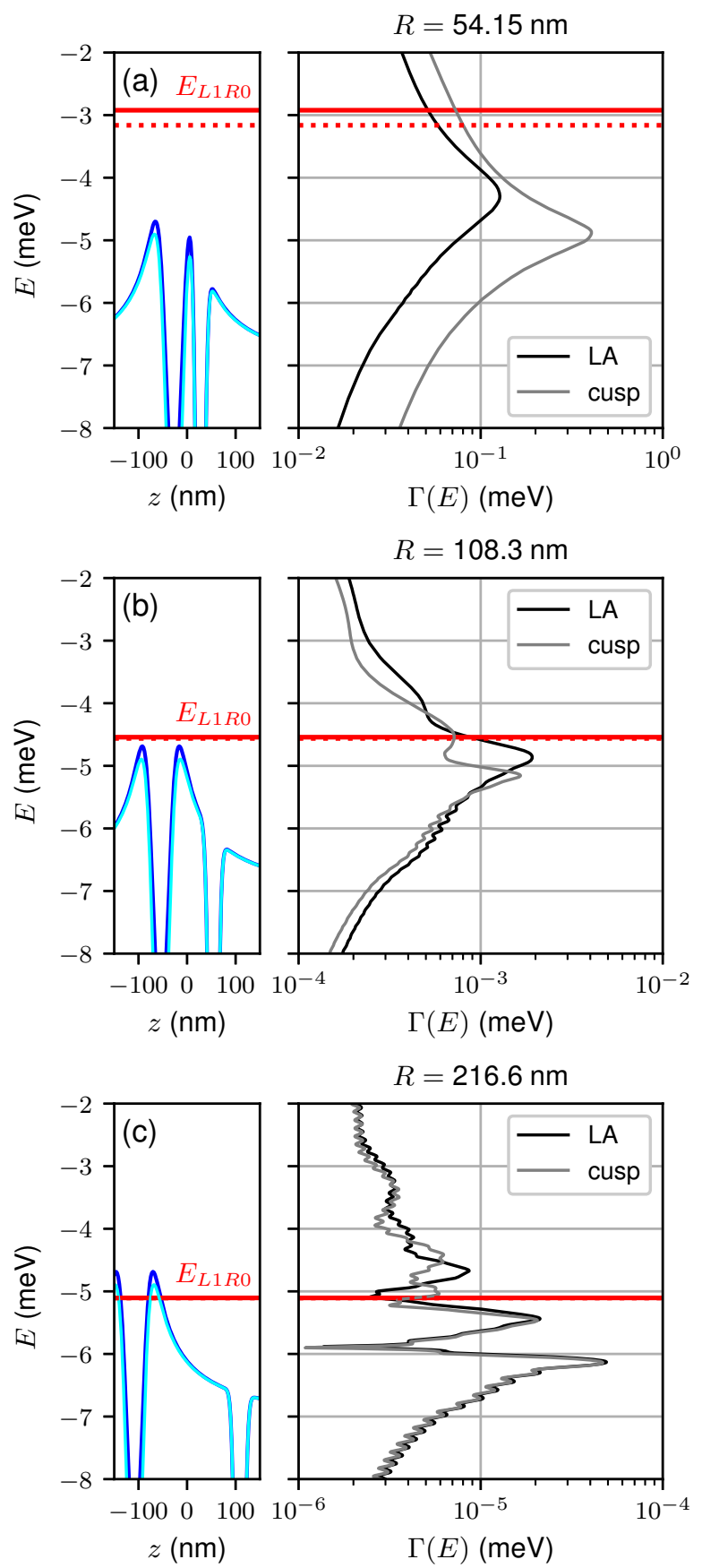

FIG. 10. Influence of the LA (dark blue) and cusp (light blue) regularized final effective potential surfaces $V_{L 0}$ (left panels) on the respective energy resolved ICD widths (right panels) obtained within the SAE TDFGR. The respective energies of the ICD resonances $E_{L 1 R 0}$ are denoted by red lines (LA: full, cusp: dotted). The rows (a,b,c) depict the results obtained for different inter-QD distances.

the overall qualitative agreement between the exact two-electron calculations and the single 
active electron representation is quite satisfactory for varying distances between the QDs.

\section{A. inter-QD distance dependence of ICD in PQDs}

To further explore the single active electron TDFGR approximation, we have calculated $\tilde{\Gamma}(E ; R)$ shown in figure 11 , for a range of inter-QD distances $R$ from $65 \mathrm{~nm}$ to $238 \mathrm{~nm}$. Here it is explicitly shown that the decay width depends parametrically on the inter-QD separation. Note that the actual ICD widths $\tilde{\Gamma}\left(E_{R}^{V L 1}(R) ; R\right)$ are also highlighted in figure 11 . As it was shown in Ref. ${ }^{22}$, the $R$ dependence of $\Gamma$ is a highly non-linear function, strongly

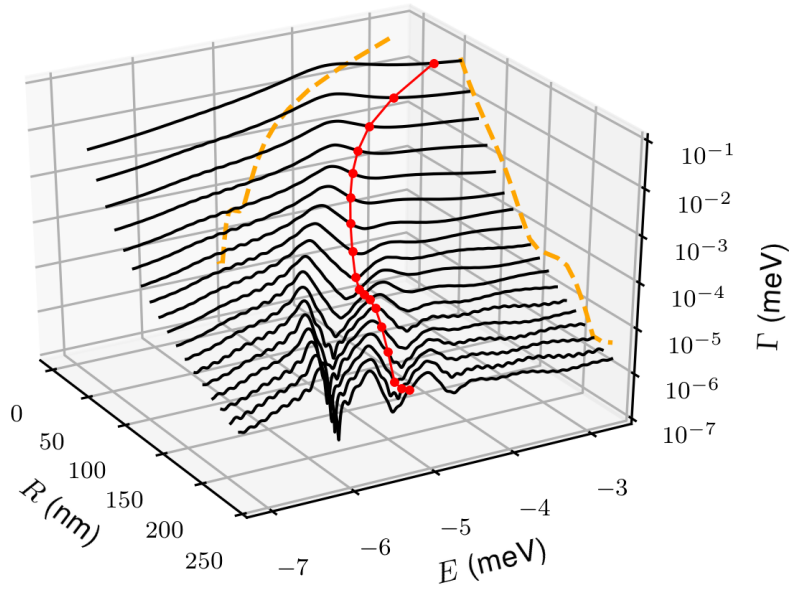

FIG. 11. inter-QD distance dependence of the energy resolved ICD widths obtained from SAE TDFGR dynamics on the $V_{L 0}$ effective potential including the LA regularized Coulomb interaction, where the red filled circles mark the widths at the respective ICD resonance energies $E_{R}^{V L 1}(R) \approx E_{\mathrm{L} 1 \mathrm{R} 0}(R)$. The projections of the function $\Gamma\left(E_{R}^{V L 1}(R), R\right)$ on the planes $\Gamma-R$ and $\Gamma-E$, respectively are represented by dashed orange lines.

oscillating around an overall $\sim R^{-6}$ behavior. The present results show that $\tilde{\Gamma}(E)$ generally decreases for larger $R$, in agreement with the decrease in the Coulomb interaction between the electrons and likewise the decay inducing potential $\hat{W}$. In addition, the overall shape of $\tilde{\Gamma}(E)$ is significantly modulated with increasing $R$. While one broad maximum is present in the relevant energy range for $R=54 \mathrm{~nm}$ to $R=87 \mathrm{~nm}$, additional minima and maxima arise for $R \geq 98 \mathrm{~nm}$. Correlating the energy $E_{R}^{V L 1}(R)$ of the ICD electron with the shape of $\tilde{\Gamma}(E)$ reveals that $\tilde{\Gamma}\left(E_{R}^{V L 1}(R) ; R\right)$ not necessary coincides with extrema for a given $R$. However, when it does so (at least approximately) the oscillatory behavior of $\tilde{\Gamma}\left(E_{R}^{V L 1}(R) ; R\right)$, similar 
to the results of Ref. .22 , is revealed.

While evaluating $\tilde{\Gamma}(E ; R)$ at the resonance energy $E_{R}^{V L 1}$ for a specific separation $R$ yields a single number (the decay rate), the power spectrum $\tilde{\Gamma}(E ; R)$ exhibits the properties of a probability distribution function. On the one hand, from the mathematical point of view, it is a positive-definite, bounded function, and it can be decomposed in a continuous and a discrete part $\frac{53}{3}$. On the other hand, this spectral density contains information on the microscopic dynamics. As the Fourier transform of the autocorrelation function, $\tilde{\Gamma}(E ; R)$ provides a measure on the importance of memory effects, that is, how long it takes the system to evolve from the initial state to the orthonormal final state. In the case of ICD in PQDs, for short inter-QD separations, the power spectrum spreads over the entire range of energies represented in figure 11 , which translates in short correlation times (i.e., short memory) and a faster decay of the resonance state. Conversely, for larger inter-QD distances, the energy range over which $\tilde{\Gamma}(E ; R)$ is non-zero gets narrower. This is a fingerprint of a slowly decaying initial state (i.e., long memory).

As the distance between the QDs becomes larger, the resonance energy lies below the height of the Coulomb barrier (see figure $10 \mathrm{c}$ ), therefore the lifetime of the resonant state increases. This trend gives rise to the following scenario. The projection on the continuum states of the active electron wavepacket, initially located around the right-hand well, spreads in both directions. While $\Phi_{c}(t)$ propagates freely to the right, the portion of the density distribution moving leftwards impinge on the energy barrier around the left QD, and it is partially scattered back to the region of the right QD (see figure 8c). This motion results in fractional revivals of $\Phi_{c}(t)$, and consequently in a more structured power spectrum $\tilde{\Gamma}(E ; R)$ (notably, the peaks and valleys displayed in figure 11.

In figure 12, we show variations of the decay widths with respect to the inter-QD distance $R$, computed using the exact two-electron dynamics and the SAE TDFGR model with effective potentials obtained from the LA regularized (equation (4), see also figure 11), and from the cusp regularized Coulomb interaction (equation (5)), respectively. The other parameters of the confining potential are chosen as stated before and held constant. Compared to the exact results, the use of effective potentials yields a faster decay in the small- $R$ regime $(R<87 \mathrm{~nm})$. Additionally, the results of the one-electron approximations do not show the maximum in the decay width around $R=65 \mathrm{~nm}$. For well-separated QDs $(R \geq 87 \mathrm{~nm})$, the agreement is remarkable between the decay rates obtained from the exact two-electron 
wavepacket propagation, and from the SEA approach using the LA regularized Coulomb potential. The oscillations of the ICD width with the increase of the spatial separation

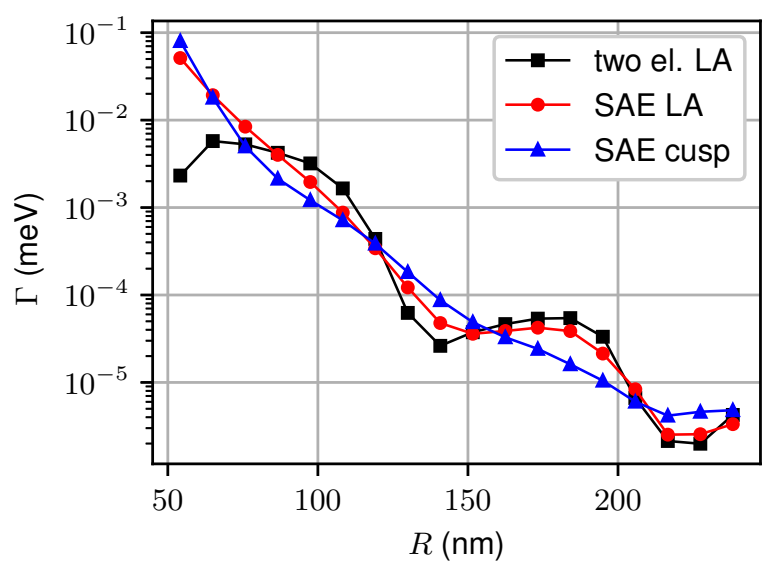

FIG. 12. Dependence of ICD widths on the inter-QD distance. Here we compare widths obtained from the exact two-electron calculation (solid squares) and from the SAE TDGFR (solid circles). Both are derived with respect to the LA regularized Coulomb interaction. SAE TDFGR results based on the cusp regularized Coulomb interaction are depicted by solid triangles. The SAE TDFGR widths are determined from the functions $\tilde{\Gamma}(E ; R)$ at the respective approximated resonance energy for a given $R$, i.e. $\tilde{\Gamma}\left(E_{R}^{V L 1}(R) ; R\right)$ is shown, where $E_{R}^{V L 1}(R) \approx E_{\mathrm{L} 1 \mathrm{R} 0}(R)$.

between the QDs is well captured by this effective potential approach.

Likewise, the general trend of the ICD widths calculated in the framework of the SAE TDFGR using the cusp regularized Coulomb interaction, equation (5) (that is, modelling the short-range electron correlations approximately using the Jastrow parameter $\alpha(R))$ is roughly piece-wise linear, with different slopes for the intervals $54 \mathrm{~nm} \leq R \leq 108 \mathrm{~nm}$ and 108 $\mathrm{nm} \leq R \leq 238 \mathrm{~nm}$. These ranges closely match the regions of rapid variation of the function $\alpha(R)$, and the plateau that follows the former. The disparate behaviour for short and large interdot separations is consistent with the rationale that for small distances between the QDs there is a non-negligible, $R$-dependent overlap between the electron densities corresponding to the bound states of each well, and different values of $\alpha$ are required in order to reproduce the effect of pair correlations. However, for well-separated QDs, the parameter $\alpha$ must account for two-body effects when the outgoing electron approaches the left QD only, and thus it is fairly independent of $R$.

Overall, the ICD widths predicted within this approach follow the global $R^{-6}$ trend of 
the two-electron benchmark, that is, the spatial oscillations of $\Gamma(R)$ are averaged out. The deviations with respect to decay rates originate (a) from the different estimations of the energy of the ICD resonance provided by the two methods and (b) from the changes in the height of the effective Coulomb barrier. For small and intermediate separations between the centres of the potential wells $(R<150 \mathrm{~nm})$, the SAE model employing the effective potential derived using the Jastrow factor (equation (5) predicts resonance energies slightly closer to the two-electron benchmark than the laterally averaged Coulomb potential (equation (4)), whereas the two approaches yield similar results for the ICD widths in this interval. At larger separations between the QDs $(R>150 \mathrm{~nm})$, the difference in the estimated resonance energies are rather small. However, these small differences translate in the aforementioned flattening of the $\tilde{\Gamma}\left(E_{R}^{V L 1}(R) ; R\right)$ function computed using the cusp regularization, due to the small differences in the effective Coulomb barrier heights. This behaviour can be summarized as follows:

- At short distances between the QDs, the electron-electron (short-range) pair correlations play a more prominent role compared to the inclusion of the lateral degrees of freedom.

- At intermediate and large separations, pair correlations decay out, and accounting for the lateral motion in the confining potential becomes respectively as important or more relevant than these correlations.

Intuitively, this picture is expected to remain valid for neighbouring cigar-shaped QDs with different structural parameters.

\section{SUMMARY}

We have presented a quantum-dynamics study of the interparticle Coulombic decay in paired quantum dots, where the decay widths (rates) of the emitted electron are evaluated using two different effective single active electron models in the framework of the timedependent Fermi golden rule. The description focuses on the outgoing particle, since it is usually the state of the emitted electron which is more amenable for experimental detection.

While quantum-dynamics simulations for multidimensional systems continue to be a challenge, the use of the Fermi golden rule enables a dimensionality reduction based on the inte- 
gration of the coordinate of the electron that remains bound. Even though the approximate time evolution of the outgoing electron density may be unrealistic for highly correlated electron dynamics, the present analysis shows that the single active electron approach provides a qualitatively correct assessment of the ICD rates for PQDs for a wide range of inter-QD separations.

The proposed approximate methodologies predict decay widths in the environs of the reference two-body calculations providing a suitable compromise between accuracy and efficiency. For small and intermediate separations between the centres of the potential wells $(R<150 \mathrm{~nm}$ ), the SAE model employing the effective potential derived using the Jastrow factor performs better than the laterally averaged Coulomb potential in the evaluation of the energy of the ICD resonance, showing that the electron-electron (short-range) pair correlations play a more prominent role compared to the inclusion of the lateral degrees of freedom. Nevertheless, at large separations, accounting for the lateral motion in the confining potential becomes more relevant than the pair correlations.

The use of an effective Coulomb interaction partially accounting for the exchange symmetry between the electrons (via the cusp condition) is shown to average out the spatial oscillations in $\Gamma(R)$ as a function of the interdot separation $R$. Since these oscillations are only present in one-dimensional systems, the decay rates predicted within this approach are expected to behave sensitively for multidimensional systems.

A major advantage of the wavepacket Fermi golden rule treatment is its applicability to a wider class of hybrid nanostructures, such as metal nanoparticle (MNP) - quantum dot systems. Nowadays, the electronic structure of metal nanoparticles is routinely modelled using density functional techniques. Nevertheless, the description of the ICD dynamics in MNP-QD systems within the framework of density functional theory requires the use of density-dependent potentials, which are not implemented at present in the MCTDH program package. Therefore, the present method can be applied to the simulation of the ICD dynamics in the target systems by integrating the states of the metal nanoparticle, and to extend the analysis to a metal nanoparticle in the vicinity of more complex structures (e.g., an array of semiconductor quantum dots). Further investigations along this direction are currently underway. 


\section{ACKNOWLEDGEMENTS}

We would like to thank Royle Pérez Castillo for exploratory calculations. A.B. and M.B. acknowledge funding by the Volkswagen foundation (Freigeist Fellowship 89525). A.M.-M. L.U.-P. and A.B. acknowledge funding by the Deutsche Forschungsgemeinschaft (BA3770/31). We acknowledge use of the compute cluster DIRAC at Helmholtz-Zentrum Berlin.

\section{REFERENCES}

${ }^{1}$ L. S. Cederbaum, J. Zobeley, and F. Tarantelli, "Giant Intermolecular Decay and Fragmentation of Clusters," Phys. Rev. Lett. 79, 4778-4781 (1997).

${ }^{2}$ V. Averbukh, P. Demekhin, P. Kolorenč, S. Scheit, S. Stoychev, A. Kuleff, Y.-C. Chiang, K. Gokhberg, S. Kopelke, N. Sisourat, and L. Cederbaum, "Interatomic electronic decay processes in singly and multiply ionized clusters," J. Electron Spectrosc. Relat. Phenom. 183, $36-47$ (2011).

${ }^{3} \mathrm{U}$. Hergenhahn, "Production of low kinetic energy electrons and energetic ion pairs by Intermolecular Coulombic Decay," Int. J. Radiat. Biol. 88, 871-883 (2012).

${ }^{4}$ T. Jahnke, "Interatomic and intermolecular Coulombic decay: the coming of age story," J. Phys. B. 48, 082001 (2015).

${ }^{5}$ R. Santra, J. Zobeley, L. S. Cederbaum, and N. Moiseyev, "Interatomic coulombic decay in van der waals clusters and impact of nuclear motion," Phys. Rev. Lett. 85, 4490-4493 (2000).

${ }^{6}$ R. Santra, J. Zobeley, and L. S. Cederbaum, "Electronic decay of valence holes in clusters and condensed matter," Phys. Rev. B 64, 245104 (2001).

${ }^{7}$ J. Zobeley, R. Santra, and L. S. Cederbaum, "Electronic decay in weakly bound heteroclusters: Energy transfer versus electron transfer," J. Chem. Phys. 115, 5076-5088 (2001).

${ }^{8}$ T. Jahnke, A. Czasch, M. S. Schöffler, S. Schössler, A. Knapp, M. Käsz, J. Titze, C. Wimmer, K. Kreidi, R. E. Grisenti, A. Staudte, O. Jagutzki, U. Hergenhahn, H. SchmidtBöcking, and R. Dörner, "Experimental observation of interatomic coulombic decay in neon dimers," Phys. Rev. Lett. 93, 163401 (2004).

${ }^{9}$ S. Marburger, O. Kugeler, U. Hergenhahn, and T. Möller, "Experimental evidence for interatomic coulombic decay in ne clusters," Phys. Rev. Lett. 90, 203401 (2003). 
${ }^{10}$ G. Öhrwall, M. Tchaplyguine, M. Lundwall, R. Feifel, H. Bergersen, T. Rander, A. Lindblad, J. Schulz, S. Peredkov, S. Barth, S. Marburger, U. Hergenhahn, S. Svensson, and O. Björneholm, "Femtosecond interatomic coulombic decay in free neon clusters: Large lifetime differences between surface and bulk," Phys. Rev. Lett. 93, 173401 (2004).

${ }^{11}$ N. Sisourat, N. V. Kryzhevoi, P. Kolorenc, S. Scheit, T. Jahnke, and L. S. Cederbaum, "Ultralong-range energy transfer by interatomic Coulombic decay in an extreme quantum system," Nature Phys. 6, 508-511 (2010).

${ }^{12}$ E. F. Aziz, N. Ottosson, M. Faubel, I. V. Hertel, and B. Winter, "Interaction between liquid water and hydroxide revealed by core-hole de-excitation," Nature 455, 89-91 (2008).

${ }^{13}$ C. P. Schwartz, S. Fatehi, R. J. Saykally, and D. Prendergast, "Importance of Electronic Relaxation for Inter-Coulombic Decay in Aqueous Systems," Phys. Rev. Lett. 105, 198102 $(2010)$.

${ }^{14}$ T. Jahnke, H. Sann, T. Havermeier, K. Kreidi, C. Stuck, M. Meckel, M. Schöffler, N. Neumann, R. Wallauer, S. Voss, A. Czasch, O. Jagutzki, A. Malakzadeh, F. Afaneh, T. Weber, H. Schmidt-Böcking, and R. Dörner, "Ultrafast energy transfer between water molecules," Nature Phys. 6, 139-142 (2010).

${ }^{15}$ S. Thürmer, M. Ončák, N. Ottosson, R. Seidel, U. Hergenhahn, S. E. Bradforth, P. Slavícek, and B. Winter, "On the nature and origin of dicationic, charge-separated species formed in liquid water on X-ray irradiation," Nature Chem. 5, 590-596 (2013), article.

${ }^{16}$ P. H. P. Harbach, M. Schneider, S. Faraji, and A. Dreuw, "Intermolecular Coulombic Decay in Biology: The Initial Electron Detachment from FADH- in DNA Photolyases," J. Phys. Chem. Lett. 4, 943-949 (2013).

${ }^{17}$ E. Surdutovich and A. Solov'yov, "Double strand breaks in DNA resulting from double ionization events," Eur. Phys. J. D 66, 206 (2012).

${ }^{18} \mathrm{~S}$. Kazandjian, J. Rist, M. Weller, F. Wiegandt, D. Aslitürk, S. Grundmann, M. Kircher, G. Nalin, D. Pitters, I. Vela Pérez, M. Waitz, G. Schiwietz, B. Griffin, J. B. Williams, R. Dörner, M. Schöffler, T. Miteva, F. Trinter, T. Jahnke, and N. Sisourat, "Frustrated Coulomb explosion of small helium clusters," Phys. Rev. A 98, 050701(R) (2018).

${ }^{19}$ M. Shcherbinin, A. C. LaForge, V. Sharma, M. Devetta, R. Richter, R. Moshammer, T. Pfeifer, and M. Mudrich, "Interatomic Coulombic decay in helium nanodroplets," Phys. Rev. A 96, 013407 (2017). 
${ }^{20}$ R. A. Wilhelm, E. Gruber, J. Schwestka, R. Kozubek, T. I. Madeira, J. P. Marques, J. Kobus, A. V. Krasheninnikov, M. Schleberger, , and F. Aumayr, "Interatomic Coulombic Decay: The Mechanism for Rapid Deexcitation of Hollow Atoms," Phys. Rev. Lett. 119, 103401 (2017).

${ }^{21}$ I. Cherkes and N. Moiseyev, "Electron relaxation in quantum dots by the interatomic Coulombic decay mechanism," Phys. Rev. B 83, 113303 (2011).

${ }^{22}$ A. Bande, K. Gokhberg, and L. S. Cederbaum, "Dynamics of interatomic Coulombic decay in quantum dots," J. Chem. Phys. 135, 144112 (2011).

${ }^{23}$ A. Bande, "Electron dynamics of interatomic Coulombic decay in quantum dots induced by a laser field," J. Chem. Phys. 138, 214104 (2013).

${ }^{24}$ P. Dolbundalchok, D. Peláez, E. F. Aziz, and A. Bande, "Geometrical control of the interatomic Coulombic decay process in quantum dots for infrared photodetectors," J. Comput. Chem. 37, 2249-2259 (2016).

${ }^{25}$ A. Haller, Y.-C. Chiang, M. Menger, E. F. Aziz, and A. Bande, "Strong field control of the interatomic Coulombic decay process in quantum dots," Chem. Phys. 482, 135 - 145 (2016).

${ }^{26}$ F. Weber, E. F. Aziz, and A. Bande, "Interdependence of ICD rates in paired quantum dots on geometry," J. Comput. Chem. 38, 2141-2150 (2017).

${ }^{27}$ T. Goldzak, L. Gantz, I. Gilary, G. Bahir, and N. Moiseyev, "Interatomic coulombic decay in two coupled quantum wells," Phys. Rev. B 91, 165312 (2015).

${ }^{28}$ T. Goldzak, L. Gantz, I. Gilary, G. Bahir, and N. Moiseyev, "Vertical currents due to interatomic coulombic decay in experiments with two coupled quantum wells," Phys. Rev. B 93, 045310 (2016).

${ }^{29}$ I. Piquero-Zulaica, J. Lobo-Checa, A. Sadeghi, Z. M. A. El-Fattah, C. Mitsui, T. Okamoto, R. Pawlak, T. Meier, A. Arnau, J. E. Ortega, J. Takeya, S. Goedecker, E. Meyer, and S. Kawai, "Precise engineering of quantum dot array coupling through their barrier widths," Nature Comm. 8, 787 (2017).

${ }^{30}$ A. Kurzmann, P. Stegmann, J. Kerski, R. Schott, A. Ludwig, A. D. Wieck, J. König, A. Lorke, and M. Geller, "Optical detection of single-electron tunneling into a semiconductor quantum dot," Phys. Rev. Lett. 122, 247403 (2019).

${ }^{31}$ A. Haller, D. Peláez, and A. Bande, "Inter-coulombic decay in laterally arranged quantum dots controlled by polarized lasers," J. Phys. Chem. C 123, 14754-14765 (2019). 
${ }^{32}$ A. Haller and A. Bande, "Favoritism of quantum dot inter-coulombic decay over direct and multi-photon ionization by laser strength and focus," J. Chem. Phys. 149, 134102 (2018).

${ }^{33}$ A. Bande, "Acoustic phonon impact on the inter-Coulombic decay process in charged quantum dot pairs," Mol. Phys. 117, 2014-2028 (2019).

${ }^{34}$ F. Langkabel, M. Lützner, and A. Bande, "Interparticle Coulombic Decay Dynamics along Single- and Double-Ionization Pathways," J. Phys. Chem. C 123, 21757-21762 (2019).

${ }^{35}$ F. M. Pont, A. Bande, and L. S. Cederbaum, "Controlled energy-selected electron capture and release in double quantum dots," Phys. Rev. B 88, 241304 (2013).

${ }^{36}$ F. M. Pont, A. Bande, and L. S. Cederbaum, "Electron-correlation driven capture and release in double quantum dots," J. Phys. Condens. Matter 28, 075301 (2016).

${ }^{37}$ A. Molle, E. R. Berikaa, F. M. Pont, and A. Bande, "Quantum size effect affecting environment assisted electron capture in quantum confinements," J. Chem. Phys. 150, 224105 $(2019)$.

${ }^{38}$ F. M. Pont, A. Molle, E. R. Berikaa, S. Bubeck, and A. Bande, "Predicting the performance of the inter-Coulombic electron capture from single-electron quantities," J. Phys. Condens. Matter 32, 065302 (2019).

${ }^{39} \mathrm{G}$. Bastard, Wave mechanics applied to semiconductor heterostructures, Monographies de physique (Les Éditions de Physique, 1988).

${ }^{40}$ P. Villarreal, S. Miret-Artés, O. Roncero, G. Delgado-Barrio, J. A. Beswick, N. Halberstadt, and R. D. Coalson, "A wave packet Golden Rule treatment of vibrational predissociation," J. Chem. Phys. 94, 4230-4233 (1991).

41 "NSM Archive - Physical Properties of Semiconductors," http://matprop.ru/GaAs_basic (accessed on November 21, 2018).

${ }^{42}$ S. Bednarek, B. Szafran, T. Chwiej, and J. Adamowski, "Effective interaction for charge carriers confined in quasi-one-dimensional nanostructures," Phys. Rev. B 68, 045328 (2003).

${ }^{43}$ W. Foulkes, L. Mitas, R. Needs, and G. Rajagopal, "Quantum Monte Carlo simulations of solids," Rev. Mod. Phys. 73, 33 (2001).

${ }^{44}$ R. Jastrow, "Many-body problem with strong forces," Phys. Rev. 98, 1479-1484 (1955).

${ }^{45}$ S. F. Boys and N. C. Handy, "The determination of energies and wavefunctions with full electronic correlation," Proc. Royal Soc. A 310, 43-61 (1969). 
${ }^{46}$ T. Kato, "On the eigenfunctions of many-particle systems in quantum mechanics," Comm. Pure Appl. Math 10, 151-177 (1957).

${ }^{47}$ D. Bressanini, G. Morosi, and M. Mella, "Robust wave function optimization procedures in quantum Monte Carlo methods," J. Chem. Phys. 116, 5345-5350 (2002).

${ }^{48}$ H.-D. Meyer, U. Manthe, and L. Cederbaum, "The multi-configurational time-dependent hartree approach," Chem. Phys. Lett. 165, 73 - 78 (1990).

${ }^{49}$ U. Manthe, H. Meyer, and L. S. Cederbaum, "Wave-packet dynamics within the multiconfiguration hartree framework: General aspects and application to nocl," J. Chem. Phys. 97, 3199-3213 (1992).

${ }^{50}$ G. A. Worth, M. H. Beck, A. Jäckle, and H.-D. Meyer, The MCTDH Package, Version 8.2, (2000). H.-D. Meyer, Version 8.3 (2002), Version 8.4 (2007). Current version: 8.4.12 (2016). See http://mctdh.uni-hd.de.

${ }^{51}$ H.-D. Meyer, F. Gatti, and G. A. Worth, eds., Multidimensional Quantum Dynamics: MCTDH Theory and Applications (Wiley-VCH, Weinheim, 2009).

${ }^{52}$ M. Beck, A. Jäckle, G. Worth, and H.-D. Meyer, "The multiconfiguration time-dependent Hartree (MCTDH) method: a highly efficient algorithm for propagating wavepackets," Phys. Rep. 324, 1 - 105 (2000).

${ }^{53}$ P. Stoica and R. Moses, Spectral analysis of signals (Prentice-Hall, New Jersey, 2005). 